\title{
On the Small Time Asymptotics of the Two-Dimensional Stochastic Navier-Stokes Equations
}

\author{
Tiange Xu \& Tusheng Zhang
}

First version: 19 December 2008

Research Report No. 26, 2008, Probability and Statistics Group

School of Mathematics, The University of Manchester 


\title{
On the small time asymptotics of the two-dimensional stochastic Navier-Stokes equations
}

\author{
Tiange $\mathrm{Xu}^{1}$, Tusheng Zhang ${ }^{1}$
}

February 1, 2008

\begin{abstract}
In this paper, we establish a small time large deviation principle (small time asymptotics) for the two-dimensional stochastic Navier-Stokes equations driven by multiplicative noise, which not only involves the study of the small noise, but also the investigation of the effect of the small, but highly nonlinear, unbounded drifts.
\end{abstract}

AMS Subject Classification: Primary 60H15 Secondary 60F10, 35Q30.

\section{Introduction}

It is well known that the two-dimensional Navier-Stokes equation with Dirichlet boundary condition describes the time evolution of an incompressible fluid and is given by

$$
\left\{\begin{array}{r}
d u-\nu \triangle u d t+(u \cdot \nabla) u d t+\nabla p d t=g d t+\sigma(t, u) d W(t), \\
(d i v u)(t, x)=0, \text { for } x \in D, t>0, \\
u(t, x)=0, \text { for } x \in \partial D, t>0, \\
u(0, x)=u_{0}(x), \text { for } x \in D,
\end{array}\right.
$$

where $D$ is a bounded domain in $\mathbb{R}^{2}$ with smooth boundary $\partial D, u(t, x) \in \mathbb{R}^{2}$ denotes the velocity field at time $t$ and position $x, p(t, x)$ denotes the pressure field, $\nu>0$ is the viscosity and $W$ is a Brownian motion on a Hilbert space. Moreover, $\sigma(t, u) d W$ is the random force field acting on the fluid and $g$ is the deterministic part of the force.

To formulate the stochastic Navier-Stokes equations, we introduce the following standard spaces:

$$
V=\left\{v \in H_{0}^{1}\left(D, \mathbb{R}^{2}\right): \nabla \cdot v=0 \text { a.e, in } D\right\},
$$

with the norm

$$
\|v\|_{V}:=\left(\int_{D}|\nabla v|^{2} d x\right)^{\frac{1}{2}}=\|v\|,
$$

and denote by $((\cdot))$ the inner product of $V . H$ is the closure of $V$ in the $L^{2}$-norm

$$
|v|_{H}:=\left(\int_{D}|v|^{2} d x\right)^{\frac{1}{2}}=|v| .
$$

The inner product on $H$ will be denoted by $(\cdot)$.

Define the operator $A$ (Stokes operator) in $H$ by the formula

$$
A u=-\nu P_{H} \triangle u, \forall u \in H^{2}\left(D, \mathbb{R}^{2}\right) \cap V,
$$

\footnotetext{
${ }^{1}$ Department of Mathematics, University of Manchester, Oxford Road, Manchester M13 9PL, England, U.K. Email: tzhang@maths.man.ac.uk
} 
where the linear operator $P_{H}$ (Helmhotz-Hodge projection) is the projection operator from $L^{2}$ to $H$, and the nonlinear operator $B$

$$
B(u, v)=P_{H}((u \cdot \nabla) v),
$$

with the notation $B(u)=B(u, u)$. Obviously the domain of $B$ requires that $(u \cdot \nabla v)$ belongs to the space $L^{2}$.

By applying the operator $P_{H}$ to each term of the above stochastic Navier-Stokes equation (NSE), we can rewrite the NSE in the following abstract form:

$$
d u(t)+A u(t) d t+B(u(t)) d t=f d t+\sigma(t, u(t)) d W(t) \quad \text { in } L^{2}\left(0, T ; V^{\prime}\right)
$$

with the initial condition

$$
u(0)=u_{0} \quad \text { in } H
$$

where $W(t)$ is a Brownian motion taking values in a Hilbert space $U$, and $V^{\prime}$ is the dual of $V$.

There exists a great amount of literature on the stochastic Navier-Stokes equation. Let us mention some of them. A good reference for stochastic Navier-Stokes equations driven by additive noise is the book [16] and the references therein. The existence and uniqueness of solutions for stochastic 2-D Navier-Stokes equations with multiplicative noise were obtained in [8] and [18]. The ergodic properties and invariant measures of the stochastic 2-D Navier-Stokes equations are studied in [9] and [12]. The small noise large deviation of the stochastic 2-D Navier-Stokes equations was established in [18] and the large deviation of occupation measures was considered in [10].

The purpose of this paper is to study the small time asymptotics (large deviations) of the two-dimensional stochastic Navier-Stokes equations driven by multiplicative noise on $C([0,1] ; H)$, which not only involves the study of the small noise, but also the investigation of the effect of the small, but highly nonlinear drift. The study of the small time asymptotics (large deviations) of finite dimensional diffusion processes were initiated by Varadhan in the influential work [21]. The small time asymptotics of infinite dimensional diffusion processes were studied in $[1],[2],[7],[13]$ and [23].

To establish the small time large deviation for the stochastic Navier-Stokes equation, as one expects, the main difficulty lies in dealing with the nonlinear term $B(u)=P_{H}((u \cdot \nabla) u)$ and the unbounded term $A u=-\nu P_{H} \triangle u$, which barely belong to $V^{\prime}$. To control $B(u)$, our idea is to show that the probability that the solution stays outside an energy ball is exponentially small so that we can restrict the solution in a sufficiently large energy ball. Another key step of obtaining the small time large deviation is to prove that the law of the solution of the Navier-Stokes equation $u(\varepsilon t)$ is exponentially equivalent to the law of the solutions of the equation:

$$
v^{\varepsilon}(t)=x+\sqrt{\varepsilon} \int_{0}^{t} \sigma\left(\varepsilon s, v^{\varepsilon}(s)\right) d W(s) .
$$

This is done through several approximations. A remarkable martingale inequality of Barlow, Davis [5], plays an important role throughout the paper. We hope that the method used for treating the non-linear term could be useful in some other study of stochastic Navier-Stokes equations.

The rest of the paper is organized as follows. In Section 2 we collect some preliminaries which are frequently used in the sequel. Section 3 is the main part of the paper, where we proved the small time large deviations for stochastic Navier Stokes equation. In Section 4, we further relax the conditions on the diffusion coefficient $\sigma(\cdot)$. 


\section{Preliminaries}

Identifying $H$ with its dual $H^{\prime}$, we consider the equation (1.1) in the framework of Gelfrand triple:

$$
V \subset H \cong H^{\prime} \subset V^{\prime} .
$$

In this way, we may consider $A$ as a bounded operator from $V$ into $V^{\prime}$. Moreover, we also denote by $<,>$, the duality between $V$ and $V^{\prime}$. Hence, for $u=\left(u_{i}\right) \in V, w=\left(w_{i}\right) \in V$, we have

$$
<A u, w>=\nu \sum_{i, j} \int_{D} \partial_{i} u_{j} \partial_{i} w_{j} d x=\nu((u, w))
$$

Introduce a trilinear form on $H \times H \times H$ by setting

$$
b(u, v, w)=\sum_{i, j}^{2} \int_{D} u_{i} \partial_{i} v_{j} w_{j} d x
$$

whenever the integral in (2.2) makes sense. In particular, if $u, v, w \in V$, then

$$
<B(u, v), w>=<(u \cdot \nabla v), w>=\sum_{i, j}^{2} \int_{D} u_{i} \partial_{i} v_{j} w_{j} d x=b(u, v, w) .
$$

By the integration by parts,

$$
b(u, v, w)=-b(u, w, v)
$$

therefore

$$
b(u, v, v)=0, \forall u, v \in V .
$$

There are some well-known estimates for $b$ (see [19] for example), which will be required in the rest of this paper and we list them here. Throughout the paper, we denote various generic positive constants by the same letter c. We have

$$
\begin{array}{r}
|b(u, v, w)| \leq c|| u\|\cdot|| v\| \cdot|| w|| \\
|b(u, v, w)| \leq c|u| \cdot|| v|| \cdot|A w| \\
|b(u, v, w)| \leq c|| u \| \cdot|v| \cdot|A w| \\
|b(u, v, w)| \leq 2|| u||^{\frac{1}{2}}|u|^{\frac{1}{2}} \cdot|| w||^{\frac{1}{2}}|w|^{\frac{1}{2}} \cdot|| v||
\end{array}
$$

for suitable $u, v, w$. Moreover, combing (2.3) and (2.8), we obtain a useful estimate as follows:

$$
|B u|_{V^{\prime}}=\sup _{\|v\| \leq 1}|b(u, u, v)|=\sup _{\|v\| \leq 1}|b(u, v, u)| \leq 2\|u\| \cdot|u| .
$$

\section{$3 \quad$ Small time asymptotics}

First, we introduce the precise assumptions on $\sigma$. Let $(\Omega, \mathcal{F}, P)$ be a complete probability space with a filtration $\mathcal{F}_{t}, t \geq 0$ that satisfies the usual conditions. Let $W(\cdot)$ be a $H$-valued Brownian motion on $(\Omega, \mathcal{F}, P)$ with the covariance operator $Q$, which is a positive, symmetric, trace class operator on $H$. Let $L_{Q}$ denote the class of linear operators $B$ such that $B Q^{\frac{1}{2}}$ is a Hilbert-Schmidt operator from $H$ to $H . L_{Q}$ is endowed with the norm $|B|_{L_{Q}}^{2}=\operatorname{trace}\left(B Q B^{*}\right)$. Let $L_{Q}^{V}$ denote the class of linear operators $\tilde{B}$ such that $\tilde{B} Q^{\frac{1}{2}}$ is a Hilbert-Schmidt operator from $H$ to $V$, endowed with the norm $|\tilde{B}|_{L_{Q}^{V}}^{2}=\operatorname{trace}\left(\tilde{B} Q \tilde{B}^{*}\right)$. Introduce 
(A.1) $E\left|u_{0}\right|^{4}<+\infty, f \in L^{4}\left(0, T ; V^{\prime}\right)$.

(A.2) There exists a constant $L$ such that $|\sigma(t, u)|_{L_{Q}}^{2} \leq L\left(1+|u|^{2}\right)$, for all $t \in(0, T)$, and all $u \in H$.

(A.3) There exists a constant $\hat{L}$ such that for $|\sigma(t, u)|_{L_{Q}^{V}}^{2} \leq \hat{L}\left(1+\|u\|^{2}\right)$, for all $t \in(0, T)$, and all $u \in V$.

(A.4) There exists a constant $K$ such that $|\sigma(t, u)-\sigma(t, v)|_{L_{Q}}^{2} \leq K|u-v|^{2}$, for all $t \in(0, T)$, and all $u, v \in H$.

(A.5) There exists a constant $\hat{K}$ such that $|\sigma(t, u)-\sigma(t, v)|_{L_{Q}^{V}}^{2} \leq \hat{K}|| u-v \|^{2}$, for all $t \in[0, T]$ and all $u, v \in V$,

It is known that (see, for example, [18]), under the assumptions (A.1),(A.2) and (A.4), the stochastic NSE

$$
\left\{\begin{array}{l}
d u(t)+A u(t) d t+B(u(t)) d t=f d t+\sigma(t, u(t)) d W(t) \\
u(0)=x \in H
\end{array}\right.
$$

has a unique strong solution $u \in L^{2}(\Omega ; C([0, T] ; H)) \cap L^{2}(\Omega \times[0, T] ; V)$, i.e.,

$$
u(t)=x-\int_{0}^{t} A u(s) d s-\int_{0}^{t} B(u(s)) d s+\int_{0}^{t} f(s) d s+\int_{0}^{t} \sigma(s, u(s)) d W(s) .
$$

Let $\varepsilon>0$, by the scaling property of the Brownian motion, it is easy to see that $u(\varepsilon t)$ coincides in law with the solution of the following equation:

$$
u^{\varepsilon}(t)=x-\varepsilon \int_{0}^{t} A u^{\varepsilon}(s) d s-\varepsilon \int_{0}^{t} B\left(u^{\varepsilon}(s)\right) d s+\varepsilon \int_{0}^{t} f(\varepsilon s) d s+\sqrt{\varepsilon} \int_{0}^{t} \sigma\left(\varepsilon s, u^{\varepsilon}(s)\right) d W(s) .
$$

Let $\mu_{x}^{\varepsilon}$ be the law of $u^{\varepsilon}(\cdot)$ on $C([0,1] ; H)$. Define a functional $I(g)$ on $C([0,1] ; H)$ by

$$
I(g)=\inf _{h \in \Gamma_{g}}\left\{\frac{1}{2} \int_{0}^{1}|\dot{h}(t)|_{H_{0}}^{2} d t\right\}
$$

where $H_{0}=Q^{\frac{1}{2}} H$ endowed with the norm $|h|_{H_{0}}^{2}=\left|Q^{-\frac{1}{2}} h\right|^{2}\left(h \in H_{0}\right)$, and

$$
\begin{aligned}
\Gamma_{g}=\left\{h \in C\left([0, T] ; H_{0}\right): \quad\right. & h(\cdot) \text { is absolutely continuous and such that } \\
& \left.g(t)=x+\int_{0}^{t} \sigma(s, g(s)) \dot{h}(s) d s, 0 \leq t \leq 1\right\} .
\end{aligned}
$$

Theorem 3.1. $\mu_{x}^{\varepsilon}$ satisfies a large deviation principle with the rate function $I(\cdot)$, that is,

(i) For any closed subset $F \subset C([0,1] ; H)$,

$$
\lim _{\varepsilon \rightarrow 0} \sup _{x_{n} \rightarrow x} \varepsilon \log \mu_{x_{n}}^{\varepsilon}(F) \leq-\inf _{g \in F}(I(g)) .
$$

(ii) For any open subset $G \subset C([0,1] ; H)$,

$$
\lim _{\varepsilon \rightarrow 0} \inf _{x_{n} \rightarrow x} \varepsilon \log \mu_{x_{n}}^{\varepsilon}(G) \geq-\inf _{g \in G}(I(g)) .
$$

Proof: Let $v^{\varepsilon}(\cdot)$ be the solution of the stochastic equation

$$
v^{\varepsilon}(t)=x+\sqrt{\varepsilon} \int_{0}^{t} \sigma\left(\varepsilon s, v^{\varepsilon}(s)\right) d W(s),
$$


and $\nu^{\varepsilon}$ be the law of $v^{\varepsilon}(\cdot)$ on the $C([0, T] ; H)$. Then by [16], we know that $\nu^{\varepsilon}$ satisfies a large deviation principle with the rate function $I(\cdot)$. Our main task is to show that two families of the probability measures $\mu^{\varepsilon}$ and $\nu^{\varepsilon}$ are exponentially equivalent, that is, for any $\delta>0$,

$$
\lim _{\varepsilon \rightarrow 0} \varepsilon \log P\left(\sup _{0 \leq t \leq 1}\left|u^{\varepsilon}(t)-v^{\varepsilon}(t)\right|^{2}>\delta\right)=-\infty .
$$

Then Theorem 3.1 follows from (3.5) and Theorem 4.2.13 in [6] for $x_{n}=x$. Slight modifications of the proof yields the general case.

Because of the non-linear form $B(, \cdot$,$) , and the unbounded operator A$, the proof of (3.5) is quite involved. We split it into several lemmas. The following result is an estimate of the probability that the solution of (3.3) leaves an energy ball.

Lemma 3.1.

$$
\lim _{M \rightarrow \infty} \sup _{0<\varepsilon \leq 1} \varepsilon \log P\left(\left(\left|u^{\varepsilon}\right|_{V}^{H}(1)\right)^{2}>M\right)=-\infty,
$$

where $\left(\left|u^{\varepsilon}\right|_{V}^{H}(1)\right)^{2}=\sup _{0 \leq t \leq 1}\left|u^{\varepsilon}(t)\right|^{2}+\varepsilon \nu \int_{0}^{1} \|\left. u^{\varepsilon}(t)\right|^{2} d t$.

Proof: Since $<B\left(u^{\varepsilon}(s)\right), u^{\varepsilon}(s)>=0$, applying Ito formula, we get

$$
\begin{aligned}
\left|u^{\varepsilon}(t)\right|^{2}= & |x|^{2}-2 \varepsilon \int_{0}^{t}<u^{\varepsilon}(s), A u^{\varepsilon}(s)>d s+2 \varepsilon \int_{0}^{t}<u^{\varepsilon}(s), f(\varepsilon s)>d s \\
& +2 \sqrt{\varepsilon} \int_{0}^{t}\left(u^{\varepsilon}(s), \sigma\left(\varepsilon s, u^{\varepsilon}(s)\right) d W(s)\right)+\varepsilon \int_{0}^{t}\left|\sigma\left(\varepsilon s, u^{\varepsilon}(s)\right)\right|_{L_{Q}}^{2} d s,
\end{aligned}
$$

that is,

$$
\begin{aligned}
\left|u^{\varepsilon}(t)\right|^{2}+2 \varepsilon \nu \int_{0}^{t} \|\left. u^{\varepsilon}(s)\right|^{2} d s= & |x|^{2}+2 \varepsilon \int_{0}^{t}<u^{\varepsilon}(s), f(\varepsilon s)>d s+2 \sqrt{\varepsilon} \int_{0}^{t}\left(u^{\varepsilon}(s), \sigma\left(\varepsilon s, u^{\varepsilon}(s)\right) d W(s)\right) \\
& +\varepsilon \int_{0}^{t}\left|\sigma\left(\varepsilon s, u^{\varepsilon}(s)\right)\right|_{L_{Q}}^{2} d s \\
= & |x|^{2}+l_{1}+l_{2}+l_{3} .
\end{aligned}
$$

For $l_{1}$, we have

$$
\left|l_{1}\right| \leq 2 \varepsilon \int_{0}^{t}\left\|u^{\varepsilon}(s)\right\| \cdot|f(\varepsilon s)|_{V^{\prime}} d s \leq \varepsilon \nu \int_{0}^{t}\left\|u^{\varepsilon}(s)\right\|^{2} d s+\frac{1}{\nu}|f|_{L^{2}\left(0, \varepsilon t ; V^{\prime}\right)}^{2} .
$$

In view of (A.2),

$$
\left|l_{3}\right| \leq \varepsilon \cdot L \int_{0}^{t}\left(1+\left|u^{\varepsilon}(s)\right|^{2}\right) d s .
$$

Putting (3.7), (3.8) and (3.9) together, it follows that

$$
\begin{aligned}
\left|u^{\varepsilon}(t)\right|^{2}+\varepsilon \nu \int_{0}^{t}|| u^{\varepsilon}||^{2} d s \leq & \left(|x|^{2}+\varepsilon L t+\frac{1}{\nu}|f|_{L^{2}\left(0, \varepsilon t ; V^{\prime}\right)}^{2}\right)+\varepsilon L \int_{0}^{t}\left|u^{\varepsilon}(s)\right|^{2} d s \\
& +2 \sqrt{\varepsilon} \int_{0}^{t}\left(u^{\varepsilon}(s), \sigma\left(\varepsilon s, u^{\varepsilon}(s)\right) d W(s)\right) .
\end{aligned}
$$

Therefore,

$$
\begin{aligned}
\left(\left|u^{\varepsilon}\right|_{V}^{H}(T)\right)^{2} \leq & 2\left(|x|^{2}+\varepsilon L T+\frac{1}{\nu}|f|_{L^{2}\left(0, \varepsilon T ; V^{\prime}\right)}^{2}\right)+2 \varepsilon L \int_{0}^{T}\left(\left|u^{\varepsilon}(s)\right|_{V}^{H}(s)\right)^{2} d s \\
& +4 \sqrt{\varepsilon} \sup _{0 \leq t \leq T}\left|\int_{0}^{t}\left(u^{\varepsilon}(s), \sigma\left(\varepsilon s, u^{\varepsilon}(s)\right) d W(s)\right)\right| .
\end{aligned}
$$


Hence, for $p \geq 2$, we have,

$$
\begin{aligned}
\left(E\left(\left|u^{\varepsilon}\right|_{V}^{H}(T)\right)^{2 p}\right)^{\frac{1}{p}} \leq & 2\left(|x|^{2}+\varepsilon L T+\frac{1}{\nu}|f|_{L^{2}\left(0, \varepsilon T ; V^{\prime}\right)}^{2}\right)+2 \varepsilon L\left(E\left(\int_{0}^{T}\left(\left|u^{\varepsilon}\right|_{V}^{H}(s)\right)^{2} d s\right)^{p}\right)^{\frac{1}{p}} \\
& +4 \sqrt{\varepsilon}\left(E\left(\sup _{0 \leq t \leq T}\left|\int_{0}^{t}\left(u^{\varepsilon}(s), \sigma\left(\varepsilon s, u^{\varepsilon}(s)\right) d W(s)\right)\right|^{p}\right)^{\frac{1}{p}}\right.
\end{aligned}
$$

To estimate the stochastic integral term, we will use the following remarkable result from [5], [4] that there exists a universal constant $c$ such that, for any $p \geq 2$ and for any continuous martingale $\left(M_{t}\right)$ with $M_{0}=0$, one has

$$
\left\|M_{t}^{*}\right\|_{p} \leq c p^{\frac{1}{2}}\left\|<M>_{t}^{\frac{1}{2}}\right\|_{p},
$$

where $M_{t}^{*}=\sup _{0 \leq s \leq t}\left|M_{s}\right|$ and $\|\cdot\|_{p}$ stands for the $L^{P}$-norm.

Using this result, we have

$$
\begin{aligned}
& 4 \sqrt{\varepsilon}\left(E\left(\sup _{0 \leq t \leq T} \int_{0}^{t}\left(u^{\varepsilon}(s), \sigma\left(\varepsilon s, u^{\varepsilon}(s)\right) d W(s)\right)\right)^{p}\right)^{\frac{1}{p}} \\
\leq & 4 c \sqrt{p \varepsilon}\left(E\left(\int_{0}^{T}\left|u^{\varepsilon}(s)\right|^{2} \cdot\left|\sigma\left(\varepsilon s, u^{\varepsilon}(s)\right)\right|_{L_{Q}}^{2} d s\right)^{\frac{p}{2}}\right)^{\frac{1}{p}} \\
\leq & 4 c \sqrt{p \varepsilon}\left(E\left(\int_{0}^{T}\left|u^{\varepsilon}(s)\right|^{2}\left(1+\left|u^{\varepsilon}(s)\right|^{2}\right) d s\right)^{\frac{p}{2}}\right)^{\frac{1}{p}} \\
\leq & 4 c \sqrt{p \varepsilon}\left[\left(E\left(\int_{0}^{T}\left(1+\left|u^{\varepsilon}(s)\right|^{2}\right)^{2} d s\right)^{\frac{p}{2}}\right)^{\frac{2}{p}}\right]^{\frac{1}{2}} \\
\leq & 4 c \sqrt{p \varepsilon}\left[\left(E\left(\int_{0}^{T}\left(1+\left|u^{\varepsilon}(s)\right|^{4}\right) d s\right)^{\frac{p}{2}}\right)^{\frac{2}{p}}\right]^{\frac{1}{2}} \\
\leq & 4 c \sqrt{p \varepsilon}\left[\int_{0}^{T} 1+\left(E\left|u^{\varepsilon}(s)\right|^{2 p}\right)^{\frac{2}{p}} d s\right]^{\frac{1}{2}}
\end{aligned}
$$

On the other hand,

$$
\left.2 \varepsilon L\left(E\left(\int_{0}^{T}\left(\left|u^{\varepsilon}\right|_{V}^{H}(s)\right)^{2} d s\right)^{p}\right)^{\frac{1}{p}} \leq 2 \varepsilon L \int_{0}^{T}\left(E\left|u^{\varepsilon}\right|_{V}^{H}(s)\right)^{2 p}\right)^{\frac{1}{p}} d s .
$$

Combing (3.10), (3.12) and (3.13), we arrive at

$$
\begin{aligned}
& \left(E\left(\left|u^{\varepsilon}\right|_{V}^{H}(T)\right)^{2 p}\right)^{\frac{2}{p}} \\
\leq & 8\left(|x|^{2}+\varepsilon L T+\frac{1}{\nu}|f|_{L^{2}\left(0, \varepsilon T ; V^{\prime}\right)}^{2}\right)^{2}+8 \varepsilon^{2} L^{2} T \int_{0}^{T}\left(E\left(\left|u^{\varepsilon}\right|_{V}^{H}(s)\right)^{2 p}\right)^{\frac{2}{p}} d s \\
& +32 c^{2} p \varepsilon T+32 c^{2} p \varepsilon \int_{0}^{T}\left(E\left(\left|u^{\varepsilon}\right|_{V}^{H}(s)\right)^{2 p}\right)^{\frac{2}{p}} d s .
\end{aligned}
$$

Applying the Gronwall inequality, we obtain

$$
\begin{aligned}
& \left(E\left(\left|u^{\varepsilon}\right|_{V}^{H}(1)\right)^{2 p}\right)^{\frac{2}{p}} \\
\leq & {\left[8\left(|x|^{2}+\varepsilon L+\frac{1}{\nu}|f|_{L^{2}\left(0, \varepsilon ; V^{\prime}\right)}^{2}\right)^{2}+32 c^{2} p \varepsilon\right] \cdot \exp \left(8 \varepsilon^{2} L^{2}+32 c^{2} p \varepsilon\right) }
\end{aligned}
$$

Since $P\left(\left(\left|u^{\varepsilon}\right|_{V}^{H}(1)\right)^{2}>M\right) \leq M^{-p} E\left(\left|u^{\varepsilon}\right|_{V}^{H}(1)\right)^{2 p}$, let $p=\frac{1}{\varepsilon}$ in (3.15) to get

$$
\begin{aligned}
& \varepsilon \log P\left(\left(\left|u^{\varepsilon}\right|_{V}^{H}(1)\right)^{2}>M\right) \\
\leq & -\log M+\log \left(E\left(\left|u^{\varepsilon}\right|_{V}^{H}(1)\right)^{2 p}\right)^{\frac{1}{p}} \\
\leq & -\log M+\log \sqrt{\left[8\left(|x|^{2}+\varepsilon L+\frac{1}{\nu}|f|_{L^{2}\left(0, \varepsilon ; V^{\prime}\right)}^{2}\right)^{2}+32 c^{2}\right]}+4 \varepsilon^{2} L^{2}+16 c^{2} .
\end{aligned}
$$


Therefore,

$$
\begin{aligned}
& \sup _{0<\varepsilon \leq 1} \varepsilon \log P\left(\left(\left|u^{\varepsilon}\right|_{V}^{H}(1)\right)^{2}>M\right) \\
\leq & -\log M+\log \sqrt{\left[8\left(|x|^{2}+L+\frac{1}{\nu}|f|_{L^{2}\left(0,1 ; V^{\prime}\right)}^{2}\right)^{2}+32 c^{2}\right]}+16 c^{2}+4 L^{2} .
\end{aligned}
$$

Letting $M \rightarrow \infty$ on both side of the above inequality, we finish the proof.

Since $V$ is dense in $H$, there exists a sequence $\left\{x_{n}\right\}_{n=1}^{\infty} \subset V$ such that

$$
\lim _{n \rightarrow+\infty}\left|x_{n}-x\right|=0 .
$$

Let $u_{n}^{\varepsilon}(\cdot)$ be the solution of $(3.3)$ with the initial value $x_{n}$. From the proof of Lemma 3.1, it follows that

$$
\lim _{M \rightarrow+\infty} \sup _{n} \sup _{0<\varepsilon \leq 1} \varepsilon \log P\left(\left(\left|u_{n}^{\varepsilon}\right|_{V}^{H}(1)\right)^{2}>M\right)=-\infty .
$$

Let $v_{n}^{\varepsilon}(\cdot)$ be the solution of (3.4) with the initial value $x_{n}$, and we have the following result.

Lemma 3.2. For any $n \in \mathbb{Z}^{+}$,

$$
\lim _{M \rightarrow \infty} \sup _{0<\varepsilon \leq 1} \varepsilon \log P\left(\sup _{0 \leq t \leq 1}\left\|v_{n}^{\varepsilon}(t)\right\|^{2}>M\right)=-\infty .
$$

Proof: Applying Itô formula to $\left\|v_{n}^{\varepsilon}(t)\right\|^{2}$, one obtains

$$
\begin{aligned}
\left\|v_{n}^{\varepsilon}(t)\right\|^{2}= & \left\|x_{n}\right\|^{2}+2 \sqrt{\varepsilon} \int_{0}^{t}\left(\left(v_{n}^{\varepsilon}(s), \sigma\left(\varepsilon s, v_{n}^{\varepsilon}(s)\right) d W(s)\right)\right) \\
& +\varepsilon \int_{0}^{t}\left|\sigma\left(\varepsilon s, v_{n}^{\varepsilon}(s)\right)\right|_{L_{Q}^{V}}^{2} d s .
\end{aligned}
$$

By (A.3) and inequality (3.11), we have

$$
\begin{aligned}
\left(E\left[\sup _{0 \leq t \leq r}\left\|v_{n}^{\varepsilon}(t)\right\|^{2 p}\right]\right)^{\frac{2}{p}} \leq & 2\left\|x_{n}\right\|^{4}+8 c \varepsilon p\left(E\left[\int_{0}^{r}\left\|v_{n}^{\varepsilon}(s)\right\|^{2}\left|\sigma\left(\varepsilon s, v_{n}^{\varepsilon}(s)\right)\right|_{L_{Q}^{V}}^{2} d s\right]^{\frac{p}{2}}\right)^{\frac{2}{p}} \\
& +4 \varepsilon^{2} \hat{L}^{2} \lambda^{2}\left(1+\int_{0}^{r}\left(E\left[\sup _{0 \leq l \leq s}\left\|v_{n}^{\varepsilon}(l)\right\|^{2 p}\right]\right)^{\frac{2}{p}} d s\right) \\
\leq & 2\left\|x_{n}\right\|^{4}+16 c \varepsilon p \hat{L}\left(r+\int_{0}^{r}\left(E\left[\sup _{0 \leq l \leq s}\left\|v_{n}^{\varepsilon}(l)\right\|^{2 p}\right]\right)^{\frac{2}{p}} d s\right) \\
& +4 \varepsilon^{2} \hat{L}^{2} r\left(r+\int_{0}^{r}\left(E\left[\sup _{0 \leq l \leq s}\left\|v_{n}^{\varepsilon}(l)\right\|^{2 p}\right]\right)^{\frac{2}{p}} d s\right) .
\end{aligned}
$$

By Gronwall inequality,

$$
\left(E\left[\sup _{0 \leq t \leq 1}\left\|v_{n}^{\varepsilon}(t)\right\|^{2 p}\right]\right)^{\frac{2}{p}} \leq\left(2\left\|x_{n}\right\|^{4}+16 c \varepsilon p \hat{L}+4 \varepsilon^{2} \hat{L}^{2}\right) e^{16 c \varepsilon p \hat{L}+4 \varepsilon^{2} \hat{L}^{2}} .
$$

The rest of the proof is the same as that of Lemma 3.1.

Lemma 3.3. For any $\delta>0$,

$$
\lim _{n \rightarrow+\infty} \sup _{0<\varepsilon \leq 1} \varepsilon \log P\left(\sup _{0 \leq t \leq 1}\left|u^{\varepsilon}(t)-u_{n}^{\varepsilon}(t)\right|^{2}>\delta\right)=-\infty .
$$


Proof: For $M>0$, define a stopping time

$$
\tau_{\varepsilon, M}=\inf \left\{t: \varepsilon \int_{0}^{t}\left\|u^{\varepsilon}(r)\right\|^{2} d r>M, \text { or }\left|u^{\varepsilon}(t)\right|^{2}>M\right\} .
$$

Clearly,

$$
\begin{aligned}
& P\left(\sup _{0 \leq t \leq 1}\left|u^{\varepsilon}(t)-u_{n}^{\varepsilon}(t)\right|^{2}>\delta,\left(\left|u^{\varepsilon}\right|_{V}^{H}(1)\right)^{2} \leq M\right) \\
\leq & P\left(\sup _{0 \leq t \leq 1}\left|u^{\varepsilon}(t)-u_{n}^{\varepsilon}(t)\right|^{2}>\delta, \tau_{\varepsilon, M} \geq 1\right) \\
\leq & P\left(\sup _{0 \leq t \leq 1 \wedge \tau_{\varepsilon, M}}\left|u^{\varepsilon}(t)-u_{n}^{\varepsilon}(t)\right|^{2}>\delta\right) .
\end{aligned}
$$

Let $\mathrm{k}$ be a positive constant. Applying Ito formula to $e^{-k \varepsilon \int_{0}^{t \wedge \tau_{\varepsilon, M}} \| u^{\varepsilon}(s)||^{2} d s}\left|u^{\varepsilon}\left(t \wedge \tau_{\varepsilon, M}\right)-u_{n}^{\varepsilon}\left(t \wedge \tau_{\varepsilon, M}\right)\right|^{2}$, we get

$$
\begin{aligned}
& e^{-k \varepsilon \int_{0}^{t \wedge \tau_{\varepsilon, M}}\left\|u^{\varepsilon}(s)\right\|^{2} d s}\left|u^{\varepsilon}\left(t \wedge \tau_{\varepsilon, M}\right)-u_{n}^{\varepsilon}\left(t \wedge \tau_{\varepsilon, M}\right)\right|^{2}+2 \varepsilon \nu \int_{0}^{t \wedge \tau_{\varepsilon, M}} e^{-\left.k \varepsilon \int_{0}^{s}\left\|u^{\varepsilon}(r)\right\|\right|^{2} d r}\left\|u^{\varepsilon}(s)-u_{n}^{\varepsilon}(s)\right\|^{2} d s \\
= & \left|x-x_{n}\right|^{2}-\left.k \varepsilon \int_{0}^{t \wedge \tau_{\varepsilon, M}} e^{-k \varepsilon \int_{0}^{s}\left\|u^{\varepsilon}(r)\right\|^{2} d r}\left\|u^{\varepsilon}(s)\right\|\right|^{2}\left|u^{\varepsilon}(s)-u_{n}^{\varepsilon}(s)\right|^{2} d s \\
& -2 \varepsilon \int_{0}^{t \wedge \tau_{\varepsilon, M}} e^{-k \varepsilon \int_{0}^{s}\left\|u^{\varepsilon}(r)\right\|^{2} d r}\left(b\left(u^{\varepsilon}(s), u^{\varepsilon}(s), u^{\varepsilon}(s)-u_{n}^{\varepsilon}(s)\right)-b\left(u_{n}^{\varepsilon}(s), u_{n}^{\varepsilon}(s), u^{\varepsilon}(s)-u_{n}^{\varepsilon}(s)\right) d s\right. \\
& +\varepsilon \int_{0}^{t \wedge \tau_{\varepsilon, M}} e^{-k \varepsilon \int_{0}^{s}\left\|u^{\varepsilon}(r)\right\|^{2} d r}\left|\sigma\left(\varepsilon s, u^{\varepsilon}(s)\right)-\sigma\left(\varepsilon s, u_{n}^{\varepsilon}(s)\right)\right|_{L_{Q}}^{2} d s \\
& +2 \sqrt{\varepsilon} \int_{0}^{t \wedge \tau_{\varepsilon, M}} e^{-k \varepsilon \int_{0}^{s}\left\|u^{\varepsilon}(r)\right\|^{2} d r}\left(u^{\varepsilon}(s)-u_{n}^{\varepsilon}(s),\left(\sigma\left(\varepsilon s, u^{\varepsilon}(s)\right)-\sigma\left(\varepsilon s, u_{n}^{\varepsilon}(s)\right)\right) d W(s)\right) .
\end{aligned}
$$

Notice that by (2.4), we have

$$
b\left(u_{n}^{\varepsilon}(t), u_{n}^{\varepsilon}(t), u^{\varepsilon}(t)-u_{n}^{\varepsilon}(t)\right)=b\left(u_{n}^{\varepsilon}(t), u^{\varepsilon}(t), u^{\varepsilon}(t)-u_{n}^{\varepsilon}(t)\right),
$$

hence by (2.9),

$$
\begin{aligned}
& \left|b\left(u^{\varepsilon}(t), u^{\varepsilon}(t), u^{\varepsilon}(t)-u_{n}^{\varepsilon}(t)\right)-b\left(u_{n}^{\varepsilon}(t), u_{n}^{\varepsilon}(t), u^{\varepsilon}(t)-u_{n}^{\varepsilon}(t)\right)\right| \\
= & \left|b\left(u^{\varepsilon}(t)-u_{n}^{\varepsilon}(t), u^{\varepsilon}(t), u^{\varepsilon}(t)-u_{n}^{\varepsilon}(t)\right)\right| \\
\leq & 2\left|u^{\varepsilon}(t)-u_{n}^{\varepsilon}(t)\right| \cdot|| u^{\varepsilon}(t) \| \cdot|| u^{\varepsilon}(t)-u_{n}^{\varepsilon}(t)|| .
\end{aligned}
$$


Therefore, by (3.21),

$$
\begin{aligned}
& e^{-k \varepsilon \int_{0}^{t \wedge \tau_{\varepsilon, M}}\left\|u^{\varepsilon}(s)\right\|^{2} d s}\left|u^{\varepsilon}\left(t \wedge \tau_{\varepsilon, M}\right)-u_{n}^{\varepsilon}\left(t \wedge \tau_{\varepsilon, M}\right)\right|^{2}+2 \varepsilon \nu \int_{0}^{t \wedge \tau_{\varepsilon, M}} e^{-k \varepsilon \int_{0}^{s}\left\|u^{\varepsilon}(r)\right\|^{2} d r}\left\|u^{\varepsilon}(s)-u_{n}^{\varepsilon}(s)\right\|^{2} d s \\
\leq & \left|x-x_{n}\right|^{2}-k \varepsilon \int_{0}^{t \wedge \tau_{\varepsilon, M}} e^{-k \varepsilon \int_{0}^{s}\left\|u^{\varepsilon}(r)\right\|^{2} d r}|| u^{\varepsilon}(s) \|^{2}\left|u^{\varepsilon}(s)-u_{n}^{\varepsilon}(s)\right|^{2} d s \\
& +4 \varepsilon \int_{0}^{t \wedge \tau_{\varepsilon, M}} e^{-k \varepsilon \int_{0}^{s}\left\|u^{\varepsilon}(r)\right\|^{2} d r}\left|u^{\varepsilon}(s)-u_{n}^{\varepsilon}(s)\right| \cdot|| u^{\varepsilon}(s)\|\cdot\| u^{\varepsilon}(s)-u_{n}^{\varepsilon}(s) \| d s \\
& +\varepsilon K \int_{0}^{t \wedge \tau_{\varepsilon, M}} e^{-k \varepsilon \int_{0}^{s}\left\|u^{\varepsilon}(r)\right\|^{2} d r}\left|u^{\varepsilon}(s)-u_{n}^{\varepsilon}(s)\right|^{2} d s \\
& +2 \sqrt{\varepsilon} \mid \int_{0}^{t \wedge \tau_{\varepsilon, M}} e^{-k \varepsilon \int_{0}^{s}\left\|u^{\varepsilon}(r)\right\|^{2} d r}\left(u^{\varepsilon}(s)-u_{n}^{\varepsilon}(s),\left(\sigma\left(\varepsilon s, u^{\varepsilon}(s)\right)-\sigma\left(\varepsilon s, u_{n}^{\varepsilon}(s)\right) d W(s)\right) \mid\right. \\
\leq & \left|x-x_{n}\right|^{2}-k \varepsilon \int_{0}^{t \wedge \tau_{\varepsilon, M}} e^{-k \varepsilon \int_{0}^{s}\left\|u^{\varepsilon}(r)\right\|^{2} d r}\left\|u^{\varepsilon}(s)\right\|^{2}\left|u^{\varepsilon}(s)-u_{n}^{\varepsilon}(s)\right|^{2} d s \\
& +\varepsilon \nu \int_{0}^{t \wedge \tau_{\varepsilon, M}} e^{-k \varepsilon \int_{0}^{s}\left\|u^{\varepsilon}(r)\right\|^{2} d r}\left\|u^{\varepsilon}(s)-u_{n}^{\varepsilon}(s)\right\|^{2} d s \\
& +\frac{4 \varepsilon}{\nu} \int_{0}^{t \wedge \tau_{\varepsilon, M}} e^{-k \varepsilon \int_{0}^{s}\left\|u^{\varepsilon}(r)\right\|^{2} d r}\left|u^{\varepsilon}(s)-u_{n}^{\varepsilon}(s)\right|^{2} \cdot\left\|u^{\varepsilon}(s)\right\|^{2} d s \\
& +\varepsilon K \int_{0}^{t \wedge \tau_{\varepsilon, M}} e^{-k \varepsilon \int_{0}^{s}\left\|u^{\varepsilon}(r)\right\|^{2} d r}\left|u^{\varepsilon}(s)-u_{n}^{\varepsilon}(s)\right|^{2} d s \\
& +2 \sqrt{\varepsilon} \mid \int_{0}^{t \wedge \tau_{\varepsilon, M}} e^{-k \varepsilon \int_{0}^{s}\left\|u^{\varepsilon}(r)\right\|^{2} d r}\left(u^{\varepsilon}(s)-u_{n}^{\varepsilon}(s),\left(\sigma\left(\varepsilon s, u^{\varepsilon}(s)\right)-\sigma\left(\varepsilon s, u_{n}^{\varepsilon}(s)\right) d W(s)\right) \mid .\right.
\end{aligned}
$$

Choosing $k>\frac{4}{\nu}$ and using (3.11), we have,

$$
\begin{aligned}
& \left.\left(E\left[\sup _{0 \leq s \leq t \wedge \tau_{\varepsilon, M}}\left(e^{-k \int_{0}^{s}\left\|u^{\varepsilon}(r)\right\| \|^{2} d r}\left|u^{\varepsilon}(s)-u_{n}^{\varepsilon}(s)\right|^{2}\right)\right)^{p}\right]\right)^{\frac{2}{p}} \\
\leq & 2\left|x-x_{n}\right|^{4}+2 \varepsilon^{2} K^{2} \int_{0}^{t}\left(E\left[\left(\sup _{0 \leq r \leq s \wedge \tau_{\varepsilon, M}} e^{-k \varepsilon \int_{0}^{r}\left\|u^{\varepsilon}(l)\right\| \|^{2} d l}\left|u^{\varepsilon}(r)-u_{n}^{\varepsilon}(r)\right|^{2}\right)^{p}\right]\right)^{\frac{2}{p}} d s \\
& +8 c \varepsilon p K^{2} \int_{0}^{t}\left(E\left[\left(\sup _{0 \leq r \leq s \wedge \tau_{\varepsilon, M}} e^{-2 k \varepsilon \int_{0}^{r}\left\|u^{\varepsilon}(l)\right\|^{2} d l}\left|u^{\varepsilon}(r)-u_{n}^{\varepsilon}(r)\right|^{4}\right)^{\frac{p}{2}}\right]\right)^{\frac{2}{p}} d s \\
\leq & 2\left|x-x_{n}\right|^{4}+2 \varepsilon^{2} K^{2} \int_{0}^{t}\left(E\left[\left(\sup _{0 \leq r \leq s \wedge \tau_{\varepsilon, M}} e^{-k \varepsilon \int_{0}^{r}\left\|u^{\varepsilon}(l)\right\| \|^{2} d l}\left|u^{\varepsilon}(r)-u_{n}^{\varepsilon}(r)\right|^{2}\right)^{p}\right]\right)^{\frac{2}{p}} d s \\
& +8 c \varepsilon p K^{2} \int_{0}^{t}\left(E\left[\left(\sup _{0 \leq r \leq s \wedge \tau_{\varepsilon, M}} e^{-k \varepsilon \int_{0}^{r}\left\|u^{\varepsilon}(l)\right\|^{2} d l}\left|u^{\varepsilon}(r)-u_{n}^{\varepsilon}(r)\right|^{2}\right)^{p}\right]\right)^{\frac{2}{p}} d s .
\end{aligned}
$$

Applying Gronwall inequality, one obtains,

$$
\begin{aligned}
& \left(E\left[\sup _{0 \leq t \leq 1 \wedge \tau_{\varepsilon, M}}\left(e^{-k \varepsilon \int_{0}^{t} \| u^{\varepsilon}(s)||^{2} d s}\left|u^{\varepsilon}(t)-u_{n}^{\varepsilon}(t)\right|^{2}\right)^{p}\right]\right)^{\frac{2}{p}} \\
\leq & 2\left|x-x_{n}\right|^{4} e^{2 \varepsilon^{2} K^{2}+8 c \varepsilon p K^{2}} .
\end{aligned}
$$


Hence,

$$
\begin{aligned}
& \left(E\left[\sup _{0 \leq t \leq 1 \wedge \tau_{\varepsilon, M}}\left(\left|u^{\varepsilon}(t)-u_{n}^{\varepsilon}(t)\right|^{2}\right)^{p}\right]\right)^{\frac{2}{p}} \\
\leq & \left(E\left[\sup _{0 \leq t \leq 1 \wedge \tau_{\varepsilon, M}}\left(e^{-k \varepsilon \int_{0}^{t}\left\|u^{\varepsilon}(s)\right\|^{2} d s}\left|u^{\varepsilon}(t)-u_{n}^{\varepsilon}(t)\right|^{2}\right)^{p} e^{k p \varepsilon \int_{0}^{1 \wedge \tau_{\varepsilon, M}}\left\|u^{\varepsilon}(s)\right\|^{2} d s}\right]\right)^{\frac{2}{p}} \\
\leq & e^{2 k M}\left(E\left[\sup _{0 \leq t \leq 1 \wedge \tau_{\varepsilon, M}}\left(e^{-k \varepsilon \int_{0}^{t}\left\|u^{\varepsilon}(s)\right\|^{2} d s}\left|u^{\varepsilon}(t)-u_{n}^{\varepsilon}(t)\right|^{2}\right)^{p}\right]\right)^{\frac{2}{p}} \\
\leq & 2 e^{2 k M}\left|x-x_{n}\right|^{4} e^{2 \varepsilon^{2} K^{2}+8 c \varepsilon p K^{2}} .
\end{aligned}
$$

Fix $\mathrm{M}$, and take $p=\frac{2}{\varepsilon}$ to get

$$
\begin{aligned}
& \sup _{0<\varepsilon \leq 1} \varepsilon \log P\left(\sup _{0 \leq t \leq 1 \wedge \tau_{\varepsilon, M}}\left|u^{\varepsilon}(t)-u_{n}^{\varepsilon}(t)\right|^{2}>\delta\right) \\
\leq & \sup _{0 \leq \varepsilon \leq 1} \varepsilon \log \frac{E\left[\sup _{0 \leq t \leq 1 \wedge \tau_{\varepsilon, M}}\left|u^{\varepsilon}(t)-u_{n}^{\varepsilon}(t)\right|^{2 p}\right]}{\delta^{p}} \\
\leq & 2 k M+2 K^{2}+16 c K^{2}-2 \log \delta+\log 2\left|x-x_{n}\right|^{4} \\
\rightarrow & -\infty, \quad \text { as } n \rightarrow+\infty .
\end{aligned}
$$

By Lemma 3.1, for any $R>0$, there exists a constant $M$ such that for any $\varepsilon \in(0,1]$, the following inequality holds,

$$
P\left(\left(\left|u^{\varepsilon}\right|_{V}^{H}(1)\right)^{2}>M\right) \leq e^{-\frac{R}{\varepsilon}}
$$

For such a $M$, by (3.20), (3.26), there exits a positive integer $N$, such that for any $n \geq N$,

$$
\sup _{0<\varepsilon \leq 1} \varepsilon \log P\left(\sup _{0 \leq t \leq 1}\left|u^{\varepsilon}(t)-u_{n}^{\varepsilon}(t)\right|^{2}>\delta,\left(\left|u^{\varepsilon}\right|_{V}^{H}(1)\right)^{2} \leq M\right) \leq-R .
$$

Putting (3.27) and (3.28) together, one sees that there exists a positive integer $N$, such that for any $n \geq N, \varepsilon \in(0,1]$

$$
P\left(\sup _{0 \leq t \leq 1}\left|u^{\varepsilon}(t)-u_{n}^{\varepsilon}(t)\right|^{2}>\delta\right) \leq 2 e^{-\frac{R}{\varepsilon}} .
$$

Since $R$ is arbitrary, the lemma follows.

We have the following similar result for $v^{\varepsilon}(\cdot)$ and $v_{n}^{\varepsilon}(\cdot)$.

Lemma 3.4. For any $\delta>0$,

$$
\lim _{n \rightarrow+\infty} \sup _{0<\varepsilon \leq 1} \varepsilon \log P\left(\sup _{0 \leq t \leq 1}\left|v^{\varepsilon}(t)-v_{n}^{\varepsilon}(t)\right|^{2}>\delta\right)=-\infty .
$$

Proof: The proof is similar to that of Lemma 3.3. Applying Ito formula to $\left|v^{\varepsilon}(t)-v_{n}^{\varepsilon}(t)\right|^{2}$, and taking (3.11),(A.4) into consideration, it follows that

$$
\begin{aligned}
& \left(E\left[\sup _{0 \leq s \leq t}\left|v^{\varepsilon}(s)-v_{n}^{\varepsilon}(s)\right|^{2 p}\right]\right)^{\frac{2}{p}} \\
\leq & 2\left|x-x_{n}\right|^{4}+\left(8 c \varepsilon p K^{2}+2 \varepsilon^{2} K^{2}\right) \int_{0}^{t}\left(E\left[\sup _{0 \leq r \leq s}\left|v^{\varepsilon}(r)-v_{n}^{\varepsilon}(r)\right|^{2 p}\right]\right)^{\frac{2}{p}} d s .
\end{aligned}
$$

Applying Gronwall inequality,

$$
\left(E\left[\sup _{0 \leq t \leq 1}\left|v^{\varepsilon}(t)-v_{n}^{\varepsilon}(t)\right|^{2 p}\right]\right)^{\frac{2}{p}} \leq 2\left|x-x_{n}\right|^{4} e^{8 c \varepsilon p K^{2}+2 \varepsilon^{2} K^{2}} .
$$

The rest proof is the same as that of Lemma 3.3. 
Lemma 3.5. For any $\delta>0$, and every positive integer $n$,

$$
\lim _{\varepsilon \rightarrow 0} \varepsilon \log P\left(\sup _{0 \leq t \leq 1}\left|u_{n}^{\varepsilon}(t)-v_{n}^{\varepsilon}(t)\right|^{2}>\delta\right)=-\infty .
$$

Proof: For $M>0$, define the following stopping times:

$$
\begin{gathered}
\tau_{\varepsilon, M}^{1, n}=\inf \left\{t \geq 0: \varepsilon \int_{0}^{t}\left\|u_{n}^{\varepsilon}(s)\right\|^{2} d s>M, \text { or }\left|u_{n}^{\varepsilon}(t)\right|^{2}>M\right\}, \\
\tau_{\varepsilon, M}^{2, n}=\inf \left\{t \geq 0:\left\|v_{n}^{\varepsilon}(t)\right\|^{2}>M\right\} .
\end{gathered}
$$

Then we have

$$
\begin{aligned}
& \left.P\left(\sup _{0 \leq t \leq 1}\left|u_{n}^{\varepsilon}(t)-v_{n}^{\varepsilon}(t)\right|^{2}>\delta,\left(\left|u_{n}^{\varepsilon}\right|_{V}^{H}(1)\right)^{2}\right) \leq M, \sup _{0 \leq t \leq 1}\left\|v_{n}^{\varepsilon}(t)\right\|^{2} \leq M\right) \\
\leq & P\left(\sup _{0 \leq t \leq 1}\left|u_{n}^{\varepsilon}(t)-v_{n}^{\varepsilon}(t)\right|^{2}>\delta, 1 \leq \tau_{\varepsilon, M}^{1, n} \wedge \tau_{\varepsilon, M}^{2, n}\right) \\
\leq & P\left(\sup _{0 \leq t \leq 1 \wedge \tau_{\varepsilon, M}^{1, n} \wedge \tau_{\varepsilon, M}^{2, n}}\left|u_{n}^{\varepsilon}(t)-v_{n}^{\varepsilon}(t)\right|^{2}>\delta\right) .
\end{aligned}
$$

Put $\tau_{\varepsilon, M}^{n}=\tau_{\varepsilon, M}^{1, n} \wedge \tau_{\varepsilon, M}^{2, n}$. Applying Itô formula to $\left|u_{n}^{\varepsilon}\left(t \wedge \tau_{\varepsilon, M}^{n}\right)-v_{n}^{\varepsilon}\left(t \wedge \tau_{\varepsilon, M}^{n}\right)\right|^{2}$, we get

$$
\begin{aligned}
& \left|u_{n}^{\varepsilon}\left(t \wedge \tau_{\varepsilon, M}^{n}\right)-v_{n}^{\varepsilon}\left(t \wedge \tau_{\varepsilon, M}^{n}\right)\right|^{2}+2 \varepsilon \nu \int_{0}^{t \wedge \tau_{\varepsilon, M}^{n}}\left\|u_{n}^{\varepsilon}(s)-v_{n}^{\varepsilon}(s)\right\|^{2} d s \\
= & -2 \varepsilon \int_{0}^{t \wedge \tau_{\varepsilon, M}^{n}}<u^{\varepsilon}(s)-v_{n}^{\varepsilon}(s), A v_{n}^{\varepsilon}(s)>d s-2 \varepsilon \int_{0}^{t \wedge \tau_{\varepsilon, M}^{n}} b\left(u_{n}^{\varepsilon}(s), u_{n}^{\varepsilon}(s), u_{n}^{\varepsilon}(s)-v_{n}^{\varepsilon}(s)\right) d s \\
& +2 \varepsilon \int_{0}^{t \wedge \tau_{\varepsilon, M}^{n}}<u_{n}^{\varepsilon}(s)-v_{n}^{\varepsilon}(s), f(\varepsilon s)>d s+\varepsilon \int_{0}^{t \wedge \tau_{\varepsilon, M}^{n}}\left|\sigma\left(\varepsilon s, u_{n}^{\varepsilon}(s)\right)-\sigma\left(\varepsilon s, v_{n}^{\varepsilon}(s)\right)\right|_{L_{Q}}^{2} d s \\
& +2 \sqrt{\varepsilon} \int_{0}^{t \wedge \tau_{\varepsilon, M}^{n}}\left(u_{n}^{\varepsilon}(s)-v_{n}^{\varepsilon}(s),\left(\sigma\left(\varepsilon s, u_{n}^{\varepsilon}(s)\right)-\sigma\left(\varepsilon s, v_{n}^{\varepsilon}(s)\right) d W(s)\right)\right. \\
= & -2 \varepsilon \int_{0}^{t \wedge \tau_{\varepsilon, M}^{n}}<u_{n}^{\varepsilon}(s)-v_{n}^{\varepsilon}(s), A v_{n}^{\varepsilon}(s)>d s-2 \varepsilon \int_{0}^{t \wedge \tau_{\varepsilon, M}^{n}} b\left(u_{n}^{\varepsilon}(s)-v_{n}^{\varepsilon}(s), u_{n}^{\varepsilon}(s), u_{n}^{\varepsilon}(s)-v_{n}^{\varepsilon}(s)\right) d s \\
& +2 \varepsilon \int_{0}^{t \wedge \tau_{\varepsilon, M}^{n}} b\left(v_{n}^{\varepsilon}(s), u_{n}^{\varepsilon}(s)-v_{n}^{\varepsilon}(s), v_{n}^{\varepsilon}(s)\right) d s+2 \varepsilon \int_{0}^{t \wedge \tau_{\varepsilon, M}^{n}}<u_{n}^{\varepsilon}(s)-v_{n}^{\varepsilon}(s), f(\varepsilon s)>d s \\
& +\varepsilon \int_{0}^{t \wedge \tau_{\varepsilon, M}^{n}}\left|\sigma\left(\varepsilon s, u_{n}^{\varepsilon}(s)\right)-\sigma\left(\varepsilon s, v_{n}^{\varepsilon}(s)\right)\right|_{L_{Q}}^{2} d s \\
& +2 \sqrt{\varepsilon} \int_{0}^{t \wedge \tau_{\varepsilon, M}^{n}}\left(u_{n}^{\varepsilon}(s)-v_{n}^{\varepsilon}(s),\left(\sigma\left(\varepsilon s, u_{n}^{\varepsilon}(s)\right)-\sigma\left(\varepsilon s, v_{n}^{\varepsilon}(s)\right) d W(s)\right) .\right.
\end{aligned}
$$

Applying the inequality

$$
2 a b \leq \frac{\nu}{4} a^{2}+\frac{4}{\nu} b^{2}, a, b \geq 0
$$


and by $(2.9)$, we have,

$$
\begin{aligned}
& \left|u_{n}^{\varepsilon}\left(t \wedge \tau_{\varepsilon, M}^{n}\right)-v_{n}^{\varepsilon}\left(t \wedge \tau_{\varepsilon, M}^{n}\right)\right|^{2}+2 \varepsilon \nu \int_{0}^{t \wedge \tau_{\varepsilon, M}^{n}}\left\|u_{n}^{\varepsilon}(s)-v_{n}^{\varepsilon}(s)\right\|^{2} d s \\
& \leq 2 \varepsilon \int_{0}^{t \wedge \tau_{\varepsilon, M}^{n}}\left|<u_{n}^{\varepsilon}(s)-v_{n}^{\varepsilon}(s), A v_{n}^{\varepsilon}(s)>\right| d s+2 \varepsilon \int_{0}^{t \wedge \tau_{\varepsilon, M}^{n}}\left|u_{n}^{\varepsilon}(s)-v_{n}^{\varepsilon}(s)\right| \cdot|| u_{n}^{\varepsilon}(s)\left\|\cdot|| u_{n}^{\varepsilon}(s)-v_{n}^{\varepsilon}(s)\right\| d s \\
& +2 \varepsilon \int_{0}^{t \wedge \tau_{\varepsilon, M}^{n}}\left|v_{n}^{\varepsilon}(s)\right| \cdot|| u_{n}^{\varepsilon}(s)-v_{n}^{\varepsilon}(s)\|\cdot\| v_{n}^{\varepsilon}(s)|| d s+2 \varepsilon \int_{0}^{t \wedge \tau_{\varepsilon, M}^{n}}\left|<u_{n}^{\varepsilon}(s)-v_{n}^{\varepsilon}(s), f(\varepsilon s)>\right| d s \\
& +\varepsilon \int_{0}^{t \wedge \tau_{\varepsilon, M}^{n}}\left|\sigma\left(\varepsilon s, u_{n}^{\varepsilon}(s)\right)-\sigma\left(\varepsilon s, v_{n}^{\varepsilon}(s)\right)\right|_{L_{Q}}^{2} d s \\
& +2 \sqrt{\varepsilon} \mid \int_{0}^{t \wedge \tau_{\varepsilon, M}^{n}}\left(u_{n}^{\varepsilon}(s)-v_{n}^{\varepsilon}(s),\left(\sigma\left(\varepsilon s, u_{n}^{\varepsilon}(s)\right)-\sigma\left(\varepsilon s, v_{n}^{\varepsilon}(s)\right) d W(s)\right) \mid\right. \\
& \leq 2 \varepsilon \int_{0}^{t \wedge \tau_{\varepsilon, M}^{n}}\left\|u_{n}^{\varepsilon}(s)-v_{n}^{\varepsilon}(s)\right\| \cdot\left\|v_{n}^{\varepsilon}(s)\right\| d s+2 \varepsilon \int_{0}^{t \wedge \tau_{\varepsilon, M}^{n}}\left|u_{n}^{\varepsilon}(s)-v_{n}^{\varepsilon}(s)\right| \cdot|| u_{n}^{\varepsilon}(s)\|\cdot\| u_{n}^{\varepsilon}(s)-v_{n}^{\varepsilon}(s) \| d s \\
& +2 \varepsilon \int_{0}^{t \wedge \tau_{\varepsilon, M}^{n}}\left|v_{n}^{\varepsilon}(s)\right| \cdot\left\|u_{n}^{\varepsilon}(s)-v_{n}^{\varepsilon}(s)\right\| \cdot\left\|v_{n}^{\varepsilon}(s)\right\| d s+2 \varepsilon \int_{0}^{t \wedge \tau_{\varepsilon, M}^{n}}\left\|u_{n}^{\varepsilon}(s)-v_{n}^{\varepsilon}(s)\right\| \cdot|f(\varepsilon s)|_{V^{\prime}} d s \\
& +\varepsilon \int_{0}^{t \wedge \tau_{\varepsilon, M}^{n}}\left|\sigma\left(\varepsilon s, u_{n}^{\varepsilon}(s)\right)-\sigma\left(\varepsilon s, v_{n}^{\varepsilon}(s)\right)\right|_{L_{Q}}^{2} d s \\
& +2 \sqrt{\varepsilon} \mid \int_{0}^{t \wedge \tau_{\varepsilon, M}^{n}}\left(u_{n}^{\varepsilon}(s)-v_{n}^{\varepsilon}(s),\left(\sigma\left(\varepsilon s, u_{n}^{\varepsilon}(s)\right)-\sigma\left(\varepsilon s, v_{n}^{\varepsilon}(s)\right) d W(s)\right) \mid .\right.
\end{aligned}
$$

Furthermore, by (A.4), we get

$$
\begin{aligned}
& \left|u_{n}^{\varepsilon}\left(t \wedge \tau_{\varepsilon, M}^{n}\right)-v_{n}^{\varepsilon}\left(t \wedge \tau_{\varepsilon, M}^{n}\right)\right|^{2}+2 \varepsilon \nu \int_{0}^{t \wedge \tau_{\varepsilon, M}^{n}}\left\|u_{n}^{\varepsilon}(s)-v_{n}^{\varepsilon}(s)\right\|^{2} d s \\
\leq & \varepsilon \nu \int_{0}^{t \wedge \tau_{\varepsilon, M}^{n}}\left\|u_{n}^{\varepsilon}(s)-v_{n}^{\varepsilon}(s)\right\|^{2} d s+\frac{4}{\nu} \varepsilon \int_{0}^{t}\left\|v_{n}^{\varepsilon}(s)\right\|^{2} d s+\frac{4}{\nu} \varepsilon \int_{0}^{t}\left|u_{n}^{\varepsilon}(s)-v_{n}^{\varepsilon}(s)\right|^{2}\left\|u_{n}^{\varepsilon}(s)\right\|^{2} d s \\
& +\frac{4}{\nu} \varepsilon \int_{0}^{t \wedge \tau_{\varepsilon, M}^{n}}|| v_{n}^{\varepsilon}(s) \|^{4} d s+\frac{4}{\nu} \int_{0}^{\varepsilon\left(t \wedge \tau_{\varepsilon, M}^{n}\right)}|f(s)|_{V^{\prime}}^{2} d s+\varepsilon K \int_{0}^{t \wedge \tau_{\varepsilon, M}^{n}}\left|u_{n}^{\varepsilon}(s)-v_{n}^{\varepsilon}(s)\right|^{2} d s \\
& +2 \sqrt{\varepsilon} \mid \int_{0}^{t \wedge \tau_{\varepsilon, M}^{n}}\left(u_{n}^{\varepsilon}(s)-v_{n}^{\varepsilon}(s),\left(\sigma\left(\varepsilon s, u_{n}^{\varepsilon}(s)\right)-\sigma\left(\varepsilon s, v_{n}^{\varepsilon}(s)\right) d W(s)\right) \mid .\right.
\end{aligned}
$$

Using the Gronwall inequality,

$$
\begin{aligned}
& \left|u_{n}^{\varepsilon}\left(t \wedge \tau_{\varepsilon, M}^{n}\right)-v_{n}^{\varepsilon}\left(t \wedge \tau_{\varepsilon, M}^{n}\right)\right|^{2} \\
\leq & \left(\frac{4}{\nu} \varepsilon \int_{0}^{t \wedge \tau_{\varepsilon, M}^{n}}\left\|v_{n}^{\varepsilon}(s)\right\|^{2} d s+\frac{4}{\nu} \varepsilon \int_{0}^{t \wedge \tau_{\varepsilon, M}^{n}}\left\|v_{n}^{\varepsilon}(s)\right\|^{4} d s+\frac{4}{\nu} \int_{0}^{\varepsilon\left(t \wedge \tau_{\varepsilon, M}^{n}\right)}|f(s)|_{V^{\prime}}^{2} d s\right. \\
& +2 \sqrt{\varepsilon} \mid \int_{0}^{t \wedge \tau_{\varepsilon, M}^{n}}\left(u_{n}^{\varepsilon}(s)-v_{n}^{\varepsilon}(s),\left(\sigma\left(\varepsilon s, u_{n}^{\varepsilon}(s)\right)-\sigma\left(\varepsilon s, v_{n}^{\varepsilon}(s)\right) d W(s)\right) \mid\right) \cdot e^{\frac{4}{\nu} \varepsilon \int_{0}^{t \wedge \tau_{\varepsilon, M}^{n}}\left\|u_{n}^{\varepsilon}(s)\right\|^{2} d s+\varepsilon K t} .
\end{aligned}
$$

By the similar techniques used in the previous lemma, (3.11), and the definition of the stopping 
time $\tau_{\varepsilon, M}^{n}$, we deduce from (3.36) that

$$
\begin{aligned}
& \left(E \sup _{0 \leq s \leq t \wedge \tau_{\varepsilon, M}^{n}}\left|u_{n}^{\varepsilon}(s)-v_{n}^{\varepsilon}(s)\right|^{2 p}\right)^{\frac{2}{p}} \\
\leq & e^{\left(\frac{8 M}{\nu}+2 \varepsilon K\right)} \cdot\left(\frac{32}{\nu^{2}} \varepsilon^{2}\left(E\left(\int_{0}^{t \wedge \tau_{\varepsilon, M}^{n}}\left\|v_{n}^{\varepsilon}(s)\right\|^{2} d s\right)^{p}\right)^{\frac{2}{p}}+\frac{32}{\nu^{2}} \varepsilon^{2}\left(E\left(\int_{0}^{t \wedge \tau_{\varepsilon, M}^{n}}\left\|v_{n}^{\varepsilon}(s)\right\|^{4} d s\right)^{p}\right)^{\frac{2}{p}}\right. \\
& \left.+\frac{32}{\nu^{2}}\left(\int_{0}^{\varepsilon}|f(s)|_{V^{\prime}}^{2} d s\right)^{2}+8 c \varepsilon p K^{2} \int_{0}^{t}\left(E \sup _{0 \leq r \leq s \wedge \tau_{\varepsilon, M}^{n}}\left|u_{n}^{\varepsilon}(r)-v_{n}^{\varepsilon}(r)\right|^{2 p}\right)^{\frac{2}{p}} d s\right) \\
\leq & e^{\left(\frac{8}{\nu} M+2 \varepsilon K\right)} \cdot\left(\frac{32}{\nu^{2}} \varepsilon^{2} M^{2}+\frac{32}{\nu^{2}} \varepsilon^{2} M^{4}+\frac{32}{\nu^{2}}\left(\int_{0}^{\varepsilon}|f(s)|_{V^{\prime}}^{2} d s\right)^{2}\right) \\
& \left.+e^{\left(\frac{8 M}{\nu}+2 \varepsilon K\right)} \cdot 8 c p \varepsilon K^{2} \int_{0}^{t}\left(E \sup _{0 \leq r \leq s \wedge \tau_{\varepsilon, M}^{n}}\left|u_{n}^{\varepsilon}(r)-v_{n}^{\varepsilon}(r)\right|^{2 p}\right)^{\frac{2}{p}} d s\right),
\end{aligned}
$$

Therefore, let $C_{\varepsilon, M}=e^{\left(\frac{8}{\nu} M+2 \varepsilon K\right)} \cdot 8 c p \varepsilon K^{2}$, we get

$$
\begin{aligned}
& \left(E \sup _{0 \leq t \leq 1 \wedge \tau_{\varepsilon, M}^{n}}\left|u_{n}^{\varepsilon}(s)-v_{n}^{\varepsilon}(s)\right|^{2 p}\right)^{\frac{2}{p}} \\
\leq & e^{\left(\frac{8}{\nu} M+2 \varepsilon K\right)} \cdot\left(\frac{32}{\nu^{2}} \varepsilon^{2} M^{2}+\frac{32}{\nu^{2}} \varepsilon^{2} M^{4}+\frac{32}{\nu^{2}}\left(\int_{0}^{\varepsilon}|f(s)|_{V^{\prime}}^{2} d s\right)^{2}\right) \cdot e^{C_{\varepsilon, M} .}
\end{aligned}
$$

From (3.16) and Lemma 3.2, we know that, for any $R>0$, there exists a M such that

$$
\begin{aligned}
& \sup _{0 \leq \varepsilon \leq 1} \varepsilon \log P\left(\left(\left|u_{n}^{\varepsilon}\right|_{V}^{H}(1)\right)^{2}>M\right) \leq-R, \\
& \sup _{0 \leq \varepsilon \leq 1} \varepsilon \log P\left(\sup _{0 \leq t \leq 1}\left\|v_{n}^{\varepsilon}\right\|^{2}>M\right) \leq-R .
\end{aligned}
$$

For such a constant $M$, let $p=\frac{2}{\varepsilon}$ in (3.38) to obtain

$$
\begin{aligned}
& \varepsilon \log P\left(\sup _{0 \leq t \leq 1}\left|u_{n}^{\varepsilon}(t)-v_{n}^{\varepsilon}(t)\right|^{2}>\delta,\left(\left|u_{n}^{\varepsilon}\right|_{V}^{H}(1)\right)^{2} \leq M, \sup _{0 \leq t \leq 1} \| v_{n}^{\varepsilon}||^{2} \leq M\right) \\
\leq & \varepsilon \log P\left(\sup _{0 \leq t \leq 1 \wedge \tau_{\varepsilon, M}^{n}}\left|u_{n}^{\varepsilon}(t)-v_{n}^{\varepsilon}(t)\right|^{2}>\delta\right) \\
\leq & \log \left(E \sup _{0 \leq t \leq 1 \wedge \tau_{\varepsilon, M}^{n}}\left|u_{n}^{\varepsilon}(t)-v_{n}^{\varepsilon}(t)\right|^{2 p}\right)^{\frac{2}{p}}-\log \delta^{2} \\
\leq & \frac{8}{\nu} M+2 \varepsilon K+\log \left(\frac{32}{\nu^{2}} \varepsilon^{2} M^{2}+\frac{32}{\nu^{2}} \varepsilon^{2} M^{4}+\frac{32}{\nu^{2}}\left(\int_{0}^{\varepsilon}|f(s)|_{V^{\prime}}^{2} d s\right)^{2}\right)+C_{M, \varepsilon}-\log \delta^{2} . \\
& \rightarrow-\infty, \text { as } \varepsilon \rightarrow 0,
\end{aligned}
$$

Thus, there exists a $\varepsilon_{0}$ such that for any $\varepsilon$ satisfying $0<\varepsilon \leq \varepsilon_{0}$,

$$
P\left(\sup _{0 \leq t \leq 1}\left|u_{n}^{\varepsilon}(t)-v_{n}^{\varepsilon}(t)\right|^{2}>\delta,\left(\left|u_{n}^{\varepsilon}\right|_{V}^{H}(1)\right)^{2} \leq M, \sup _{0 \leq t \leq 1}\left\|v_{n}^{\varepsilon}\right\|^{2} \leq M\right) \leq e^{-\frac{R}{\varepsilon}}
$$

Putting (3.39), (3.40) and (3.42) together, we see that there exists a constant $\varepsilon_{0}$ such that for any $\varepsilon \in\left(0, \varepsilon_{0}\right]$,

$$
P\left(\sup _{0 \leq t \leq 1}\left|u_{n}^{\varepsilon}(t)-v_{n}^{\varepsilon}(t)\right|^{2}>\delta\right) \leq 3 e^{-\frac{R}{\varepsilon}} .
$$

Since $R$ is arbitrary, the proof is finished. 
The proof of (3.5).

By Lemma 3.3 and Lemma 3.4, we have for any $R>0$, there exists a $N_{0}$ satisfying

$$
P\left(\sup _{0 \leq t \leq 1}\left|u^{\varepsilon}(t)-u_{N_{0}}^{\varepsilon}(t)\right|^{2}>\delta\right) \leq e^{-\frac{R}{\varepsilon}} \quad \text { for any } \varepsilon \in(0,1],
$$

and

$$
P\left(\sup _{0 \leq t \leq 1}\left|v^{\varepsilon}(t)-v_{N_{0}}^{\varepsilon}(t)\right|^{2}>\delta\right) \leq e^{-\frac{R}{\varepsilon}} \quad \text { for any } \varepsilon \in(0,1] .
$$

In view of Lemma 3.5 , for such $N_{0}$, there exists a $\varepsilon_{0}$ such that for any $\varepsilon \in\left(0, \varepsilon_{0}\right]$

$$
P\left(\sup _{0 \leq t \leq 1}\left|u_{N_{0}}^{\varepsilon}(t)-v_{N_{0}}^{\varepsilon}(t)\right|>\delta\right) \leq e^{-\frac{R}{\varepsilon}} .
$$

Thus, for any $\varepsilon \in\left(0, \varepsilon_{0}\right]$,

$$
P\left(\sup _{0 \leq t \leq 1}\left|u^{\varepsilon}(t)-v^{\varepsilon}(t)\right|>\delta\right) \leq 3 e^{-\frac{R}{\varepsilon}} .
$$

Since $R$ is arbitrary, we conclude that

$$
\lim _{\varepsilon \rightarrow 0} \varepsilon \log P\left(\sup _{0 \leq t \leq 1}\left|u^{\varepsilon}(t)-v^{\varepsilon}(t)\right|^{2}>\delta\right)=-\infty .
$$

\section{Further Results}

In this section, instead of assuming (A.3) and (A.5), we suppose that there exists a sequence of mappings $\left\{\sigma^{m}(\cdot)\right\}_{m=1}^{\infty}$ which satisfy

(A.6) There exists a constant $C_{1}(m)$, which may depend on $m$, such that for all $u \in V$, $\left|\sigma^{m}(t, u)\right|_{L_{Q}^{V}}^{2} \leq C_{1}(m)\left(1+\|u\|^{2}\right)$ for all $t \in[0, T]$.

(A.7) There exists a constant $C_{2}$ such that for all $u \in H$ and $m$, $\left|\sigma^{m}(t, u)\right|_{L_{Q}}^{2} \leq C_{2}\left(1+|u|^{2}\right)$ for all $t \in[0, T]$.

(A.8) There exists a constant $C_{3}(m)$, which may depend on $m$, such that for all $u, v \in V$, $\left|\sigma^{m}(t, u)-\sigma^{m}(t, v)\right|_{L_{Q}^{V}}^{2} \leq C_{3}(m)\|u-v\|^{2}$ for all $t \in[0, T]$.

(A.9) There exists a constant $C_{4}$ such that for all $u, v \in H$ and $m$, $\left|\sigma^{m}(t, u)-\sigma^{m}(t, v)\right|_{L_{Q}}^{2} \leq C_{4}|u-v|^{2}$ for all $t \in[0, T]$.

(A.10) $\left|\sigma^{m}(t, u)-\sigma(t, u)\right|_{L_{Q}}^{2} \rightarrow 0$ as $m \rightarrow+\infty$, uniformly on the bounded subsets of $[0, \infty) \times H$.

Theorem 4.1. Under the assumptions (A.1),(A.2),(A.4) and (A.6)-(A.10), the large deviation principle stated in Theorem 3.1 still holds for $\mu_{x}^{\varepsilon}$.

Under the assumptions (A.6)-(A.10), the stochastic equations

$$
d u^{\varepsilon, m}(t)+\varepsilon A u^{\varepsilon, m}(t) d t+\varepsilon B\left(u^{\varepsilon, m}(t)\right) d t=\varepsilon f(\varepsilon t) d t+\sqrt{\varepsilon} \sigma_{\lambda}^{m}\left(\varepsilon t, u^{\varepsilon, m}\right) d W(t)
$$

and

$$
d v^{\varepsilon, m}(t)=\sqrt{\varepsilon} \sigma\left(\varepsilon t, v^{\varepsilon, m}(g)\right) d W(t)
$$

admit unique strong solutions. We denote by $u_{n}^{\varepsilon, m}$ and $v_{n}^{\varepsilon, m}$ the solution of the above stochastic equations respectively with the same initial value as $u_{n}^{\varepsilon}$ and $v_{n}^{\varepsilon}$ in Section 3 .

In order to show Theorem 4.1, arguing as in the proof of Theorem 3.1, it is sufficient to prove 
(1) For any $n$ and $\delta>0$,

$$
\lim _{m \rightarrow \infty} \sup _{0<\varepsilon \leq 1} \varepsilon \log P\left(\sup _{0 \leq t \leq 1}\left|u_{n}^{\varepsilon}(t)-u_{n}^{\varepsilon, m}(t)\right|^{2}>\delta\right)=-\infty .
$$

(2) For any $n$ and $\delta>0$,

$$
\lim _{m \rightarrow \infty} \sup _{0<\varepsilon \leq 1} \varepsilon \log P\left(\sup _{0 \leq t \leq 1}\left|v_{n}^{\varepsilon}(t)-v_{n}^{\varepsilon, m}(t)\right|^{2}>\delta\right)=-\infty .
$$

(3) For any $n, m$ and $\delta>0$,

$$
\lim _{\varepsilon \rightarrow 0} \varepsilon \log P\left(\sup _{0 \leq t \leq 1}\left|u_{n}^{\varepsilon, m}(t)-v_{n}^{\varepsilon, m}(t)\right|^{2}>\delta\right)=-\infty .
$$

The Proof of (4.1). Similar to the proof of Lemma 3.3, for any fixed $n$, define the stopping time

$$
\tau_{\varepsilon, M}^{(n)}=\inf \left\{t \geq 0, \varepsilon \int_{0}^{t}\left\|u_{n}^{\varepsilon}(s)\right\|^{2} d s>M, \text { or }\left|u_{n}^{\varepsilon}(t)\right|^{2}>M\right\} .
$$

Applying Itô formula to $e^{-k \varepsilon \int_{0}^{t \wedge \tau_{\varepsilon, M}^{(n)}}}|| u_{n}^{\varepsilon}(s) \|^{2} d s\left|u_{n}^{\varepsilon}\left(t \wedge \tau_{\varepsilon, M}^{(n)}\right)-u_{n}^{\varepsilon, m}\left(t \wedge \tau_{\varepsilon, M}^{(n)}\right)\right|^{2}$ yields

$$
\begin{aligned}
& e^{-k \varepsilon \int_{0}^{t \wedge \tau_{\varepsilon, M}^{(n)}}\left\|u_{n}^{\varepsilon}(s)\right\|^{2} d s}\left|u_{n}^{\varepsilon}\left(t \wedge \tau_{\varepsilon, M}^{(n)}\right)-u_{n}^{\varepsilon, m}\left(t \wedge \tau_{\varepsilon, M}^{(n)}\right)\right|^{2}+2 \varepsilon \nu \int_{0}^{t \wedge \tau_{\varepsilon, M}^{(n)}} e^{-k \varepsilon \int_{0}^{s}\left\|u_{n}^{\varepsilon}(r)\right\|^{2} d r}\left\|u_{n}^{\varepsilon}(s)-u_{n}^{\varepsilon, m}(s)\right\|^{2} d s \\
\leq & -k \varepsilon \int_{0}^{t \wedge \tau_{\varepsilon, M}^{(n)}} e^{-k \varepsilon \int_{0}^{s}\left\|u_{n}^{\varepsilon}(r)\right\|^{2} d r}|| u_{n}^{\varepsilon}(s) \|^{2}\left|u_{n}^{\varepsilon}(s)-u_{n}^{\varepsilon, m}(s)\right|^{2} d s \\
& +\varepsilon \nu \int_{0}^{t \wedge \tau_{\varepsilon, M}^{(n)}} e^{-k \varepsilon \int_{0}^{s}\left\|u_{n}^{\varepsilon}(r)\right\|^{2} d r}|| u_{n}^{\varepsilon}(s)-u_{n}^{\varepsilon, m}(s) \|^{2} d s \\
& +\frac{\varepsilon}{\nu} \int_{0}^{t \wedge \tau_{\varepsilon, M}^{(n)}} e^{-k \varepsilon \int_{0}^{s}\left\|u_{n}^{\varepsilon}(r)\right\|^{2} d r}\left|u_{n}^{\varepsilon}(s)-u_{n}^{\varepsilon, m}(s)\right|^{2} \cdot\left\|u_{n}^{\varepsilon}(s)\right\|^{2} d s \\
& +2 \varepsilon \int_{0}^{t \wedge \tau_{\varepsilon, M}^{(n)}} e^{-k \varepsilon \int_{0}^{s}\left\|u_{n}^{\varepsilon}(r)\right\|^{2} d r}\left|\sigma\left(\varepsilon s, u_{n}^{\varepsilon}(s)\right)-\sigma^{m}\left(\varepsilon s, u_{n}^{\varepsilon}(s)\right)\right|_{L_{Q}}^{2} d s \\
& +2 \varepsilon \int_{0}^{t \wedge \tau_{\varepsilon, M}^{(n)}} e^{-k \varepsilon \int_{0}^{s}\left\|u_{n}^{\varepsilon}(r)\right\|^{2} d r}\left|\sigma^{m}\left(\varepsilon s, u_{n}^{\varepsilon}(s)\right)-\sigma^{m}\left(\varepsilon s, u_{n}^{\varepsilon, m}(s)\right)\right|_{L_{Q}}^{2} d s \\
& +2 \sqrt{\varepsilon} \int_{0}^{t \wedge \tau_{\varepsilon, M}^{(n)}} e^{-k \varepsilon \int_{0}^{s}\left\|u_{n}^{\varepsilon}(r)\right\|^{2} d r}\left(u_{n}^{\varepsilon}(s)-u_{n}^{\varepsilon, m}(s), \sigma\left(\varepsilon s, u_{n}^{\varepsilon}(s)\right)-\sigma^{m}\left(\varepsilon s, u_{n}^{\varepsilon}(s)\right) d W(s)\right) \\
& +2 \sqrt{\varepsilon} \int_{0}^{t \wedge \tau_{\varepsilon, M}^{(n)}} e^{-k \varepsilon \int_{0}^{s}\left\|u_{n}^{\varepsilon}(r)\right\|^{2} d r}\left(u_{n}^{\varepsilon}(s)-u_{n}^{\varepsilon, m}(s), \sigma^{m}\left(\varepsilon s, u_{n}^{\varepsilon}(s)\right)-\sigma^{m}\left(\varepsilon s, u_{n}^{\varepsilon, m}(s)\right) d W(s)\right)
\end{aligned}
$$


Taking $k>\frac{1}{\nu}$ and using (3.11), we obtain

$$
\begin{aligned}
& \left(E\left[\left(\sup _{0 \leq r \leq t \wedge \tau_{\varepsilon, M}^{(n)}} e^{-k \varepsilon \int_{0}^{r}\left\|u_{n}^{\varepsilon}(s)\right\|^{2} d s}\left|u_{n}^{\varepsilon}(r)-u_{n}^{\varepsilon, m}(r)\right|^{2}\right)^{p}\right]\right)^{\frac{2}{p}} \\
\leq & 8 \varepsilon^{2} \int_{0}^{t} \mid \sigma\left(\varepsilon\left(s \wedge \tau_{\varepsilon, M}^{(n)}\right), u_{n}^{\varepsilon}\left(s \wedge \tau_{\varepsilon, M}^{(n)}\right)\right)-\sigma^{m}\left(\varepsilon\left(s \wedge \tau_{\varepsilon, M}^{(n)}\right),\left.u_{n}^{\varepsilon}\left(\left(s \wedge \tau_{\varepsilon, M}^{(n)}\right)\right)\right|_{L_{Q, \infty}} ^{2} d s\right. \\
& +8 \varepsilon^{2} C_{4}^{2} \int_{0}^{t}\left(E\left[\left(\sup _{0 \leq r \leq s \wedge \tau_{\varepsilon, M}^{(n)}} e^{-k \varepsilon \int_{0}^{r}\left\|u_{n}^{\varepsilon}(l)\right\|^{2} d l}\left|u_{n}^{\varepsilon}(r)-u_{n}^{\varepsilon, m}(r)\right|^{2}\right)^{p}\right]\right)^{\frac{2}{p}} d s \\
+ & 4 c \varepsilon p C_{4}^{2} \int_{0}^{t}\left(E\left[\left(\sup _{0 \leq r \leq s \wedge \tau_{\varepsilon, M}^{(n)}} e^{-k \varepsilon \int_{0}^{r}\left\|u_{n}^{\varepsilon}(l) \mid\right\|^{2} d l}\left|u_{n}^{\varepsilon}(r)-u_{n}^{\varepsilon, m}(r)\right|^{2}\right)^{p}\right]\right)^{\frac{2}{p}} d s \\
+ & 4 c \varepsilon p C_{4}^{2} \int_{0}^{t} \mid \sigma\left(\varepsilon\left(s \wedge \tau_{\varepsilon, M}^{(n)}\right), u_{n}^{\varepsilon}\left(s \wedge \tau_{\varepsilon, M}^{(n)}\right)\right)-\sigma^{m}\left(\varepsilon\left(s \wedge \tau_{\varepsilon, M}^{(n)}\right),\left.u_{n}^{\varepsilon}\left(\left(s \wedge \tau_{\varepsilon, M}^{(n)}\right)\right)\right|_{L_{Q, \infty}} ^{2} d s\right. \\
+ & 8 c \varepsilon p C_{4}^{2} \int_{0}^{t}\left(E\left[\left(\sup _{0 \leq r \leq s \wedge \tau_{\varepsilon, M}^{(n)}} e^{-k \varepsilon \int_{0}^{r}\left\|u_{n}^{\varepsilon}(l) \mid\right\|^{2} d l}\left|u_{n}^{\varepsilon}(r)-u_{n}^{\varepsilon, m}(r)\right|^{2}\right)^{p}\right]\right)^{\frac{2}{p}} d s,
\end{aligned}
$$

where $|\cdot|_{L_{Q, \infty}}$ denotes the essential upper bound of $|\cdot|_{L_{Q}}$ with respect to $\omega$.

By Gronwall inequality, we deduce that

$$
\begin{aligned}
& \left(E\left[\left(\sup _{0 \leq t \leq 1 \wedge \tau_{\varepsilon, M}^{(n)}} e^{-k \varepsilon \int_{0}^{t}\left\|u_{n}^{\varepsilon}(s)\right\|^{2} d s}\left|u_{n}^{\varepsilon}(t)-u_{n}^{\varepsilon, m}(t)\right|^{2}\right)^{p}\right]\right)^{\frac{2}{p}} \\
\leq & \left(8 \varepsilon^{2}+4 c \varepsilon p C_{4}^{2}\right) \int_{0}^{1} \mid \sigma\left(\varepsilon\left(s \wedge \tau_{\varepsilon, M}^{(n)}\right), u_{n}^{\varepsilon}\left(s \wedge \tau_{\varepsilon, M}^{(n)}\right)\right)-\sigma^{m}\left(\varepsilon\left(s \wedge \tau_{\varepsilon, M}^{(n)}\right),\left.u_{n}^{\varepsilon}\left(\left(s \wedge \tau_{\varepsilon, M}^{(n)}\right)\right)\right|_{L_{Q, \infty}} ^{2} d s\right. \\
& \cdot e^{8 \varepsilon^{2} C_{4}^{2}+12 c \varepsilon p C_{4}^{2}} .
\end{aligned}
$$

Therefore,

$$
\begin{aligned}
& \left.\left(E\left[\sup _{0 \leq t \leq 1 \wedge \tau_{\varepsilon, M}^{(n)}}\left|u_{n}^{\varepsilon}(t)-u_{n}^{\varepsilon, m}(t)\right|^{2}\right)^{p}\right]\right)^{\frac{2}{p}} \\
\leq & e^{2 k M}\left(8 \varepsilon^{2}+4 c \varepsilon p C_{4}^{2}\right) \int_{0}^{1} \mid \sigma\left(\varepsilon\left(s \wedge \tau_{\varepsilon, M}^{(n)}\right), u_{n}^{\varepsilon}\left(s \wedge \tau_{\varepsilon, M}^{(n)}\right)\right)-\sigma^{m}\left(\varepsilon\left(s \wedge \tau_{\varepsilon, M}^{(n)}\right),\left.u_{n}^{\varepsilon}\left(\left(s \wedge \tau_{\varepsilon, M}^{(n)}\right)\right)\right|_{L_{Q, \infty}} ^{2} d s\right. \\
& \cdot e^{8 \varepsilon^{2} C_{4}^{2}+12 c \varepsilon p C_{4}^{2}} .
\end{aligned}
$$

Taking $p=\frac{2}{\varepsilon}$, one obtains

$$
\begin{aligned}
& \sup _{0<\varepsilon \leq 1} \varepsilon \log P\left(\sup _{0 \leq t \leq 1 \wedge \tau_{\varepsilon, M}^{(n)}}\left|u_{n}^{\varepsilon}(t)-u_{n}^{\varepsilon, m}(t)\right|^{2}>\delta\right) \\
\leq & \sup _{0<\varepsilon \leq 1} \log \left(E\left(\sup _{0 \leq t \leq 1 \wedge \tau_{\varepsilon, M}^{(n)}}\left|u_{n}^{\varepsilon}(t)-u_{n}^{\varepsilon, m}(t)\right|^{2}\right)^{p}\right)^{\frac{2}{p}}-2 \log \delta \\
\leq & \left(2 k M+8 C_{4}^{2}+12 c C_{4}^{2}\right)+\log \left(8+4 c C_{4}^{2}\right)-2 \log \delta \\
+ & \log \sup _{0 \leq t \leq 1,0<\varepsilon \leq 1} \sup _{0<\varepsilon} \mid \sigma\left(\varepsilon\left(t \wedge \tau_{\varepsilon, M}^{(n)}\right), u_{n}^{\varepsilon}\left(t \wedge \tau_{\varepsilon, M}^{(n)}\right)\right)-\sigma^{m}\left(\varepsilon\left(t \wedge \tau_{\varepsilon, M}^{(n)}\right),\left.u_{n}^{\varepsilon}\left(\left(t \wedge \tau_{\varepsilon, M}^{(n)}\right)\right)\right|_{L_{Q, \infty}} ^{2}\right.
\end{aligned}
$$

Since

$$
\sup _{0 \leq t \leq 1} \sup _{0<\varepsilon \leq 1}\left|u_{n}^{\varepsilon}\left(t \wedge \tau_{\varepsilon, M}^{(n)}\right)\right| \leq M, \quad P-a . s
$$


hence, by (A.10), it follows that

$$
\lim _{m \rightarrow+\infty} \sup _{0 \leq t \leq 1,0<\varepsilon \leq 1} \sup _{0<} \mid \sigma_{\lambda}\left(\varepsilon\left(t \wedge \tau_{\varepsilon, M}^{(n)}\right), u_{n}^{\varepsilon}\left(t \wedge \tau_{\varepsilon, M}^{(n)}\right)\right)-\sigma_{\lambda}^{m}\left(\varepsilon\left(t \wedge \tau_{\varepsilon, M}^{(n)}\right),\left.u_{n}^{\varepsilon}\left(\left(t \wedge \tau_{\varepsilon, M}^{(n)}\right)\right)\right|_{L_{Q, \infty}} ^{2}=0 .\right.
$$

Therefore, since $M$ is arbitrary, by the same argument as in the proof of Lemma 3.3, (4.1) follows.

The Proof of (4.2). The proof is similar to that of (3.32). Notice that by the same proof of Lemma 3.1, we have for any $n$ and $\delta>0$,

$$
\lim _{M \rightarrow+\infty} \sup _{0<\varepsilon \leq 1} P\left(\sup _{0 \leq t \leq 1}\left|v_{n}^{\varepsilon}(t)\right|^{2}>\delta\right)=-\infty
$$

Define, for $M>0$,

$$
\bar{\tau}_{\varepsilon, M}^{(n)}=\inf \left\{t \geq 0:\left|v_{n}^{\varepsilon}(t)\right|^{2}>M\right\}
$$

Similar to (4.5), we have

$$
\begin{aligned}
& \left(E\left[\sup _{0 \leq s \leq t \wedge \bar{\tau}_{\varepsilon, M}^{(n)}}\left|v_{n}^{\varepsilon}(s)-v_{n}^{\varepsilon, m}(s)\right|^{2 p}\right]\right)^{\frac{2}{p}} \\
\leq & 8 \varepsilon^{2} \int_{0}^{t} \mid \sigma\left(\varepsilon\left(s \wedge \bar{\tau}_{\varepsilon, M}^{(n)}\right), v_{n}^{\varepsilon}\left(s \wedge \bar{\tau}_{\varepsilon, M}^{(n)}\right)\right)-\sigma^{m}\left(\varepsilon\left(s \wedge \bar{\tau}_{\varepsilon, M}^{(n)}\right),\left.v_{n}^{\varepsilon}\left(\left(s \wedge \bar{\tau}_{\varepsilon, M}^{(n)}\right)\right)\right|_{L_{Q, \infty}} ^{2} d s\right. \\
& +8 \varepsilon^{2} C_{4}^{2} \int_{0}^{t}\left(E\left[\left(\sup _{0 \leq r \leq s \wedge \bar{\tau}_{\varepsilon, M}^{(n)}}\left|v_{n}^{\varepsilon}(r)-v_{n}^{\varepsilon, m}(r)\right|^{2}\right)^{p}\right]\right)^{\frac{2}{p}} d s \\
+ & 4 c \varepsilon p C_{4}^{2} \int_{0}^{t}\left(E\left[\left(\sup _{0 \leq r \leq s \wedge \bar{\tau}_{\varepsilon, M}^{(n)}}\left|v_{n}^{\varepsilon}(r)-v_{n}^{\varepsilon, m}(r)\right|^{2}\right)^{p}\right]\right)^{\frac{2}{p}} d s \\
+ & 4 c \varepsilon p C_{4}^{2} \int_{0}^{t} \mid \sigma\left(\varepsilon\left(s \wedge \bar{\tau}_{\varepsilon, M}^{(n)}\right), v_{n}^{\varepsilon}\left(s \wedge \tau_{\varepsilon, M}^{(n)}\right)\right)-\sigma^{m}\left(\varepsilon\left(s \wedge \bar{\tau}_{\varepsilon, M}^{(n)}\right),\left.v_{n}^{\varepsilon}\left(\left(s \wedge \bar{\tau}_{\varepsilon, M}^{(n)}\right)\right)\right|_{L_{Q, \infty}} ^{2} d s\right. \\
+ & 8 c \varepsilon p C_{4}^{2} \int_{0}^{t}\left(E\left[\left(\sup _{0 \leq r \leq s \wedge \bar{\tau}_{\varepsilon, M}^{(n)}}\left|v_{n}^{\varepsilon}(r)-v_{n}^{\varepsilon, m}(r)\right|^{2}\right)^{p}\right]\right)^{\frac{2}{p}} d s .
\end{aligned}
$$

The rest of the proof is the same as that of (4.1).

The Proof of (4.3). The procedure of the proof is exactly the same as that of Lemma 3.5. Just in this situation, we need to show, for any $n, m$

$$
\lim _{M \rightarrow+\infty} \sup _{0<\varepsilon \leq 1} \varepsilon \log P\left(\left(\left|u_{n}^{\varepsilon, m}\right|_{V}^{H}(1)\right)^{2}>M\right)=-\infty,
$$

and

$$
\lim _{M \rightarrow+\infty} \sup _{0<\varepsilon \leq 1} \varepsilon \log P\left(\sup _{0 \leq t \leq 1}\left\|v_{n}^{\varepsilon, m}(t)\right\|^{2}>M\right)=-\infty .
$$

The above results can be obtained as in the proof of Lemma 3.1 and Lemma 3.2.

\section{References}

[1] Aida, S.; Kawabi, H.: Short time asymptotics of a certain infinite dimensional diffusion process. Stochastic analysis and related topics, VII(Kusadasi, 1998), 77-124,Progr. Probab.,48, Birhasuser Boston, Boston, MA,2001. 
[2] Aida, S.; Zhang, T.S.: On the small time asymptotics of diffusion processes on path groups. Potential Anal. 16(2002),no.1,67-78.

[3] Azencott, R.: Grandes déviations et applications. Ecole d'Eté de Probabilitiés de Saint-Flour VIII,1978. Lecture Notes in Math. 779 1-176.Springer, New York, 1980.

[4] Barlow, M.T.; Yor, M.: Semi-martingale inequalities via the Garsia-Rodemich-Rumsey Lemma, and applications to local time. J.Funt.Analysis 49(1982),198-229.

[5] Davis, B: On the $L^{p}$-norms of stochastic integrals and other martingales. Duke Math.J. 43(1976),697-704.

[6] Dembo, A.; Zeitouni, O.: Large Deviations Techniques. Jones and Bartlett, Boston(1993).

[7] Fang, S.Z.; Zhang, T.S.: On the small time behavior of Ornstein-Uhlenbeck processes with unbounded linear drifts. Probab. Theory Related Fields. 114(1999),no.4,487-504.

[8] Flandoli, F.; Gatarek, D.: Martingale and stationary solution for stochastic Navier-Stokes equations. Probab. Theory Related Fields, 102(1995), 367-391.

[9] Flandoli, F.: Dissipativity and invariant measures for stochastic Navier-Stokes euqtions, Nonlinear Differential Equations Appl. 1(1994), 403-426.

[10] Gourcy, M.: A large deviation principle for 2D stochastic Navier-Stokes equation. Stochastic Process. Appl. 117(2007), no.7, 904-927.

[11] Gyöngy, I.; Krylov, N.: Existence of strong solutions for Itô stochastic equations via approximations.Probab.Theory Related Fields 105(1996), no.2,143-158.

[12] Hairer, M.; Mattingly, J.C.: Ergodicity of the 2-D Navier-Stokes equation with degenrate stochastic forcing. Ann. of Math.(2)164(2006), no.3,993-1032.

[13] Hino, M.; Ramirez, J.: Small-time Gaussian behaviour of symmetric diffusion semigroup. Ann. Probab. 31(2003), no.3, 1254-1295.

[14] Menadli, J.B.; Sritharan, S.S: Stochastic 2-D Navier-Stokes Equation. Appl.Math Optim $46(2002), 31-53$.

[15] Mikulevicius, R.; Rozovskii, B.L.: Global $L_{2}$-solutions of stochastic Navier-Stokes equations.Ann.Prbab.33(2005), no1,137-176.

[16] Da Prato, G.; Zabczyk, J.: Stochastic Equations in Infinite Dimensions. Cambridge Univ. Press(1992).

[17] Da Prato, G.; Zabczyk, J.: Ergodicity for infinite Dimenisional Systems, Cambridge Univ.Press, Cambridge,1996.

[18] Sritharan, S.S; Sundar, P.: Large deviation for the two dimensional Navier-Stokes equations with multiplicative noise. Stochastic Processes and their application, 116(2006),1636-1659.

[19] Teman, R.: Navier-Stokes Equations and Nonlinear Functional Analysis. Society for industrial and applied mathematics,1983.

[20] Teman, R.: Navier-Stokes Equations, Theory and Numercial Analysis. North-Holland, Amsterdam, 1984. 
[21] Varadhan, S.R.S: Diffusion processes in small time intervals. Comm.Pure.Appl.Math. 20(1967), 659-685.

[22] Walsh, J.B.: An introduction to stochastic partial differential equations. École d'Été de Probabilités de Saint-Flour XIV-1984. Lecture Notes in Mathematics 1180(1986), 265-437.

[23] Zhang, T.S.: On the small time asymptotics of diffusion processes on Hilbert spaces. Ann.Probab. 28(2000), no.2, 537-557.

[24] Zhang, T.S.: Large deviations for stochastic nonlinear beam equations.J.Func.Anal. 248(2007), no.1,175-201. 